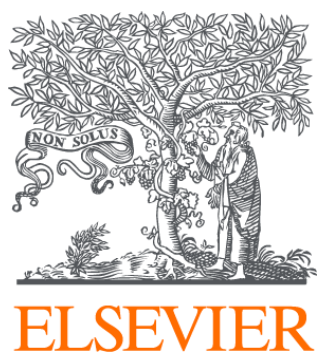

Since January 2020 Elsevier has created a COVID-19 resource centre with free information in English and Mandarin on the novel coronavirus COVID-

19. The COVID-19 resource centre is hosted on Elsevier Connect, the company's public news and information website.

Elsevier hereby grants permission to make all its COVID-19-related research that is available on the COVID-19 resource centre - including this research content - immediately available in PubMed Central and other publicly funded repositories, such as the WHO COVID database with rights for unrestricted research re-use and analyses in any form or by any means with acknowledgement of the original source. These permissions are granted for free by Elsevier for as long as the COVID-19 resource centre remains active. 


\title{
Biological responses to COVID-19: Insights from physiological and blood biomarker profiles
}

\author{
Rosita Zakeri $^{\mathrm{a}, \mathrm{b}, 1}$, Andrew Pickles ${ }^{\mathrm{c}, \mathrm{d}, 1}$, Ewan Carr ${ }^{\mathrm{c}}$, Daniel M. Bean ${ }^{\mathrm{c}, \mathrm{e}}$, Kevin O'Gallagher ${ }^{\mathrm{a}}$, \\ Zeljko Kraljewic ${ }^{c}$, Tom Searle ${ }^{\mathrm{c}, \mathrm{d}}$, Anthony Shek ${ }^{\mathrm{f}}$, James B Galloway ${ }^{\mathrm{g}}$, James T.H. Teo ${ }^{\mathrm{b}, \mathrm{f}}$, \\ Ajay M. Shah ${ }^{\mathrm{a}, \mathrm{b}}$, Richard J.B. Dobson ${ }^{\mathrm{c}, \mathrm{d}, \mathrm{h}, \mathrm{i}}$, Rebecca Bendayan ${ }^{\mathrm{c}, \mathrm{d}, *}$ \\ ${ }^{a}$ King's College London British Heart Foundation Centre of Excellence, School of Cardiovascular Medicine E' Sciences, London, SE5 9NU, UK \\ ${ }^{\mathrm{b}}$ King's College Hospital NHS Foundation Trust, London, UK \\ ${ }^{\mathrm{c}}$ Department of Biostatistics and Health Informatics, Institute of Psychiatry, Psychology and Neuroscience, King's College London, London, UK \\ d NIHR Biomedical Research Centre at South London and Maudsley NHS Foundation Trust and King's College London, London, UK \\ e Health Data Research UK London, University College London, London, UK \\ ${ }^{\mathrm{f}}$ Department of Clinical Neuroscience, Institute of Psychiatry, Psychology and Neuroscience, King's College London, London, UK \\ ${ }^{\mathrm{g}}$ Centre for Rheumatic Diseases, King's College London, London, UK \\ ${ }^{\mathrm{h}}$ Institute of Health Informatics, University College London, London, UK \\ ${ }^{\mathrm{i}}$ NIHR Biomedical Research Centre at University College London Hospitals NHS Foundation Trust, London, UK
}

\section{A R T I C L E I N F O}

\section{Article history:}

Received 29 October 2020

Accepted 26 January 2021

Available online 3 February 2021

\section{Keywords:}

Biomarkers

Classes

Inflammation

SARS-CoV-2

\begin{abstract}
A B S T R A C T
Background: Understanding the spectrum and course of biological responses to coronavirus disease 2019 (COVID-19) may have important therapeutic implications. We sought to characterise biological responses among patients hospitalised with severe COVID-19 based on serial, routinely collected, physiological and blood biomarker values.

Methods and findings: We performed a retrospective cohort study of 1335 patients hospitalised with laboratory-confirmed COVID-19 (median age 70 years, $56 \%$ male), between 1st March and 30th April 2020. Latent profile analysis was performed on serial physiological and blood biomarkers. Patient characteristics, comorbidities and rates of death and admission to intensive care, were compared between the latent classes. A five class solution provided the best fit. Class 1 "Typical response" exhibited a moderately elevated and rising C-reactive protein (CRP), stable lymphopaenia, and the lowest rates of 14-day adverse outcomes. Class 2 "Rapid hyperinflammatory response" comprised older patients, with higher admission white cell and neutrophil counts, which declined over time, accompanied by a very high and rising CRP and platelet count, and exibited the highest mortality risk. Class 3 "Progressive inflammatory response" was similar to the typical response except for a higher and rising CRP, though similar mortality rate. Class 4 "Inflammatory response with kidney injury" had prominent lymphopaenia, moderately elevated (and rising) CRP, and severe renal failure. Class 5 "Hyperinflammatory response with kidney injury" comprised older patients, with a very high and rising CRP, and severe renal failure that attenuated over time. Physiological measures did not substantially vary between classes at baseline or early admission.

Conclusions and relevance: Our identification of five distinct classes of biomarker profiles provides empirical evidence for heterogeneous biological responses to COVID-19. Early hyperinflammatory responses and kidney injury may signify unique pathophysiology that requires targeted therapy.
\end{abstract}

(c) 2021 Published by Elsevier Masson SAS.

\footnotetext{
Abbreviations: ARDS, Acute respiratory distress syndrome; BAME, Black and Minority Ethnic groups; CKD, Chronic kidney disease; COPD, Chronic obstructive pulmonary

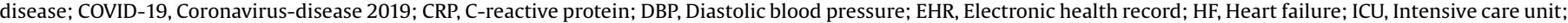

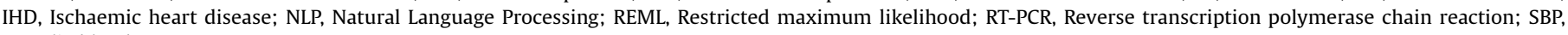
Systolic blood pressure.

* Corresponding author at: Department of Biostatistics \& Health Informatics SGDP Centre, IoPPN, Box PO 80, De Crespigny Park, Denmark Hill, London, SE5 8AF, UK.

E-mail address: rebecca.bendayan@kcl.ac.uk (R. Bendayan).

1 These authors contributed equally.
} 


\section{Introduction}

Since March 23rd, 2020, when coronavirus disease 2019 (COVID-19) was declared a global pandemic by the World Health Organization, healthcare services worldwide have faced unprecedented rates of morbidity and mortality. Although most individuals with COVID-19 experience a mild illness, a significant minority develop severe disease requiring hospitalisation. Within this group, a small subset progress to critical disease with acute respiratory distress syndrome (ARDS), requiring invasive mechanical ventilation and organ support. Older age, male sex and preexisting comorbidities have been proposed as risk factors for severe COVID-19 and poor outcomes, but the mechanisms underlying these associations remain unclear [1-3].

In practice, symptoms and physiological observations, such as persistent fever, high respiratory rate and escalating oxygen requirements identify patients who have progressive disease. However, among patients admitted to hospital, specific blood biomarker profiles have also been associated with poor outcomes, for example lymphopaenia, neutrophilia, and raised inflammatory markers, such as C-reactive protein (CRP) [4,5]. Previous studies have examined baseline (admission) biomarker levels, which may incompletely describe the subsequent clinical course and have been limited by sample size [6]. Analysis of serial measures of blood and physiological markers in large samples may highlight earlier signs of accelerated progression and provide additional insights into the biological processes that lead to adverse outcomes. Furthermore, in non-COVID related ARDS, patient subgroups with different biological responses have been shown to predict treatment effects [7].

Our aim was to examine whether different patterns of biological responses are evident among patients admitted to hospital with COVID-19. To address this aim, we: (1) examined individual patient-level physiological and blood biomarkers values at admission and their trajectories within their first 14 days of admission; (2) characterised and described subgroups of patients with distinct biological responses; and (3) examined the association between subgroups of biological response and the associated risk of critical disease (i.e. transfer to an intensive care unit [ICU] or death)

\section{Methods}

\section{Study population and study design}

We studied consecutive adults (aged $>18$ years) admitted to a multi-site acute NHS Hospital in London (UK) with laboratoryconfirmed COVID-19 between 1st March and 30th April 2020. COVID-19 was confirmed by reverse transcription polymerase chain reaction (RT-PCR) for SARS-CoV2 in oral or nasopharyngeal swabs. This project was conducted under London South East Research Ethics Committee (reference 18/LO/2048) approval granted to the King's Electronic Records Research Interface (KERRI); specific work on COVID-19 research was reviewed with expert patient input on a virtual committee with Caldicott Guardian oversight. The study adhered to the principles of the UK Data Protection Act 2018, UK National Health Service (NHS) information governance requirements, and the Declaration of Helsinki.

\section{Data extraction and processing}

Patients' demographics, clinical characteristics, laboratory results, and vital signs were retrieved and analysed in near-real time from structured and unstructured components of the electronic health record (EHR), using natural language processing
(NLP) informatics tools belonging to the CogStack/MedCAT ecosystem [8,9]. Data extraction and processing have been previously described [10] and more details are in Supplemental methods.

\section{Socio-demographics and health comorbidities}

We collected data on demographic and clinical variables putatively associated with COVID-19, including age, sex, comorbid diseases (asthma, chronic obstructive pulmonary disease [COPD], hypertension, diabetes, chronic kidney disease [CKD], ischaemic heart disease [IHD] and heart failure [HF]). Comorbidities were considered present if reported at any time up to the day of admission from the NLP pipieline. Self-reported ethnicity was classified as White or Black and Minority Ethnic groups (BAME; comprising Black, Asian, Mixed and any other non-White ethnicity). Ethnicity profiles was obtained from administrative health records (which is reconciled at every clinical encounter with the NHS Spine's Personal Demographics Service); ethnic categories are defined as per standard categories from the Office of National Statistics, United Kingdom. This ethnicity is selfdeclared and is identical to submissions to statutory public health reporting.

\section{Physiological parameters}

We examined respiratory rate (breaths per minute), oxygen saturation $\left(\mathrm{O}_{2}\right.$ sat; \%), systolic and diastolic blood pressure (SBP and DBP; $\mathrm{mmHg}$ ), heart rate (beats/min), and temperature $\left({ }^{\circ} \mathrm{C}\right)$.

\section{Blood parameters}

Blood biomarkers were selected to represent a diverse range of biological processes from those routinely obtained at or shortly after admission during clinical care. Markers with inadequate coverage over all patients and time, such as troponin, ferritin, or Ddimer, were not included. The final selection comprised nine markers: haematological indices (haemoglobin $[\mathrm{g} / \mathrm{L}]$, platelet count [plt; $\left.\times 10^{9} / \mathrm{L}\right]$ ), infection response markers (white blood cell count $\left[\mathrm{wbc}, \mathrm{x} 10^{9} / \mathrm{L}\right]$, lymphocyte count $\left[\times 10^{9} / \mathrm{L}\right]$, neutrophil count $\left[\mathrm{x} 10^{9} / \mathrm{L}\right]$ ), renal function (urea $[\mathrm{mmol} / \mathrm{L}]$, creatinine $[\mu \mathrm{mol} / \mathrm{L}]$ ), inflammation (C-reactive protein [crp; $\mathrm{mg} / \mathrm{L}]$ ) and liver synthetic function (albumin $[\mathrm{g} / \mathrm{L}]$ ). Normal values based on local assays are detailed in the Supplemental methods. Acute kidney injury was defined according to the Kidney Disease: Improving Global Outcomes definition [11].

\section{Statistical analyses}

To characterise individual biological responses, we examined patient-level physiological and blood biomarker values at admission and their trajectories within the first 14 days of admission (Objective 1), using linear mixed models. Non-linear models were estimated for each marker using restricted maximum likelihood (REML) and empirical Bayes' estimates of the random intercept and slopes. Trajectories for each marker were modelled using three piecewise slopes (0-2 days, 2-7 days, and 7-14 days) and unstructured covariance matrix. The intervals were chosen using statistical and clinical criteria described in more detail in suplemental methods section.

To classify patients based on their baseline levels and rates of change in physiological and blood biomarkers (Objective 2), latent profile models were estimated by maximum likelihood. Patients were classified on the basis of their estimated initial level and slope (rate of change) over the first two days since admission of each biomarker (see Supplemental methods for more details). 
The optimal number of latent classes was chosen based on the Integration Classification Likelihood fit statistic, that combines the Bayesian Information Criterion and entropy (McLachlan and Peel, 2000), and prevalence and interpretability of the derived classes. Demographic characteristics and pre-existing conditions of the patients assigned to each class were compared using Kruskal-Wallis and Pearson chi-square tests (objective 3 ). Class differences in the estimated biomarker levels at baseline and their change over the first two days were compared using regression t-tests.

Finally, to examine the association between subgroups and risk of in-hospital death or critical disease (i.e. transfer to ICU or death; Objective 4), we performed a multinomial logit model for the competing outcomes of ICU admission and death. Independent models were estimated for (i) outcomes within the first two days; (ii) outcomes within days 3-14. Adjustments included age, sex, ethnicity and pre-existing comorbidities. Sensitivity analyses are described in Supplemental methods. All methods were performed in accordance with the relevant guidelines and regulations.

\section{Results}

Between 1st March and 30th April 2020, 1398 patients were admitted with laboratory-confirmed COVID19 (median (IQR) age 70 (58-83) years, $56 \%$ male, $39 \%$ BAME). Characteristics of the study population are shown in the first column of Table 1 . In total, 1335 patients had serial blood biomarker data available, and were included in the latent class analyses. Excluded patients were marginally younger and had a high rate of early ICU admission.

We characterized the trajectories of the physiological and blood biomarkers for the included sample within the first 14 days of admission (Supplemental Results and Supplemental Figures 1-5). Baseline values and estimated trajectories were then used to identify subgroups.
Latent profile models: identifying subgroups of COVID-19 patients

A 5-class solution provided the optimal grouping (additional details are provided in the Supplemental results). Overall, physiological measures were similar across all 5 classes, both at baseline (intercept) and 2-day rate of change (slopes, Table 2) and are therefore not described further in detail. All classes exhibited elevated CRP levels which further increased over time. Classes 2 and 5 showed the highest CRP levels at baseline and Class 1 the lowest. The fastest rate of increase in CRP after admission was found in Class 2 and the slowest in Class 5 (Figs. 1 and 2). Differences in other blood biomarkers observed between classes are displayed in Table 2, Figs. 1 and Supplemental Figures 6 and 7.

Class $1(38 \%)$ represented the typical COVID-19 biological response in our cohort and was assigned as the reference group for comparisons. These patients exhibited lymphopaenia, moderately elevated CRP and mild anaemia (by standard haemoglobin cut-offs for men). Within the first two days the lymphocyte count remained stable, CRP increased ( $\mathrm{p}<0.001$ vs baseline), and haemoglobin decreased $(p<0.001)$. Class 1 patients were $56 \%$ male, $36 \%$ nonWhite ethnicity and their most common comorbidities were hypertension (49\%) and diabetes (33\%). Class 1 had the lowest rate of death or ICU admission within the first two days of admission and days 3-14 (3\% and $17 \%$, respectively).

Class 2 (9\%, rapid hyperinflammatory response) was characterised by prominent markers of infection response (high white cell count, neutrophilia, markedly elevated CRP), anaemia (by male-specific haemoglobin cut-offs), elevated urea and hypoalbiminaemia (Table 2). Compared with the typical COVID-19 patient response (i.e. Class 1 ), there were several markers suggesting a more severe infection response at baseline (evidenced by higher white cell count, higher neutrophil count, and higher CRP; Table 2) but also a higher platelet count (median $279 \times 10^{9} / \mathrm{L}$ [IQR 201, 393] for Class 2 versus $186 \times 10^{9} / \mathrm{L}$ [IQR 145,234$]$ for Class $1, \mathrm{p}<0.001$ ). Lymphocyte count was not significantly different from Class 1

Table 1

Baseline characteristics across COVID-19 subgroups identified using latent profile analysis.

\begin{tabular}{|c|c|c|c|c|c|c|c|}
\hline $\begin{array}{l}\text { Variable } \\
\mathrm{N}(\%)\end{array}$ & $\begin{array}{l}\text { Total } \\
1335\end{array}$ & $\begin{array}{l}\text { Class } 1 \\
511(38 \%)\end{array}$ & $\begin{array}{l}\text { Class } 2 \\
123(9 \%)\end{array}$ & $\begin{array}{l}\text { Class } 3 \\
497(37 \%)\end{array}$ & $\begin{array}{l}\text { Class } 4 \\
80(6 \%)\end{array}$ & $\begin{array}{l}\text { Class } 5 \\
124(9 \%)\end{array}$ & Class p-value* \\
\hline \multicolumn{8}{|l|}{ Demographics } \\
\hline Age in years (IQR) & $70(58-83)$ & $68(55-83)$ & $73(62-86)$ & $68(56-82)$ & $69(59-81)$ & $78(70-88)$ & $\mathrm{p}<.001$ \\
\hline Male & $754(56)$ & $285(56)$ & $64(52)$ & $264(53)$ & $54(68)$ & $87(70)$ & $\mathrm{p}=.002$ \\
\hline BAME ethnicity $(n=999)$ & $392(39)$ & $129(36)$ & $23(24)$ & $157(42)$ & $42(66)$ & $41(39)$ & $\mathrm{p}<.001$ \\
\hline \multicolumn{8}{|l|}{ Comorbidities } \\
\hline Asthma & $184(14)$ & $63(12)$ & $16(13)$ & $83(17)$ & $12(15)$ & $10(8)$ & $\mathrm{p}=.090$ \\
\hline COPD & $148(11)$ & $57(11)$ & $18(15)$ & $44(9)$ & $6(8)$ & 23 (19) & $\mathrm{p}<.016$ \\
\hline Hypertension & $732(55)$ & $251(49)$ & $64(52)$ & $262(53)$ & $65(81)$ & $90(73)$ & $\mathrm{p}<.001$ \\
\hline IHD & $195(15)$ & $64(13)$ & $19(15)$ & $67(13)$ & $17(21)$ & $28(23)$ & $\mathrm{p}=.021$ \\
\hline Heart failure & $125(9)$ & $47(9)$ & $12(10)$ & $36(7)$ & $15(19)$ & $15(12)$ & $\mathrm{p}=.017$ \\
\hline Diabetes & $463(35)$ & $167(33)$ & $37(30)$ & $153(31)$ & $51(64)$ & $55(44)$ & $\mathrm{p}<.001$ \\
\hline CKD & $242(18)$ & $56(11)$ & $19(15)$ & $51(10)$ & $55(69)$ & $61(49)$ & $\mathrm{p}<.001$ \\
\hline \multicolumn{8}{|c|}{ Symptom duration prior to admission } \\
\hline Days $(\mathrm{n}=1144)(\mathrm{IQR})$ & $5(1-7)$ & $4(1-7)$ & $3(1-7)$ & $5(2-8)$ & $3(1-7)$ & $2(1-5)$ & $\mathrm{p}=.007$ \\
\hline Symptoms after admission & $191(14)$ & $80(16)$ & $24(20)$ & $64(13)$ & $10(13)$ & $13(10)$ & $\mathrm{p}=.199$ \\
\hline \multicolumn{8}{|l|}{ In-hospital outcome } \\
\hline Death & $273(20)$ & $71(14)$ & $47(38)$ & $87(18)$ & $17(21)$ & $51(41)$ & \\
\hline Within $0-2$ days & $45(3)$ & $6(1)$ & $10(8)$ & $17(3)$ & $2(3)$ & $10(8)$ & $\mathrm{p}<.001$ \\
\hline Within $3-14$ days $(n=1251)$ & $228(18)$ & $65(13)$ & $37(34)$ & $70(15)$ & $15(20)$ & $41(37)$ & $\mathrm{p}<.001$ \\
\hline ICU & $105(8)$ & $26(5)$ & $11(9)$ & $55(11)$ & $9(11)$ & $4(3)$ & \\
\hline Within $0-2$ days & $39(3)$ & $8(2)$ & $5(4)$ & $20(4)$ & $4(5)$ & $2(2)$ & $\mathrm{p}=.082$ \\
\hline Within $3-14$ days $(n=1251)$ & $66(5)$ & $18(4)$ & $6(6)$ & $35(8)$ & $5(7)$ & $2(2)$ & $\mathrm{p}=.036$ \\
\hline \multicolumn{8}{|l|}{ Death or ICU (composite) } \\
\hline Within $0-2$ days & $84(6)$ & $14(3)$ & $15(12)$ & $37(7)$ & $6(7)$ & $12(10)$ & $\mathrm{p}<.001$ \\
\hline Within $3-14$ days $(n=1251)$ & $294(24)$ & $83(17)$ & $43(40)$ & $105(23)$ & $20(29)$ & $43(38)$ & $\mathrm{p}<.001$ \\
\hline
\end{tabular}

Data are presented as $\mathrm{n}(\%)$ or median (IQR).

BAME, Black and Minority Ethnic group; CKD, chronic kidney disease; COPD, chronic obstructive pulmonary disease;

Descriptive statistics: whole sample and by latent class.

* p-value from difference of mean Kruskall-Wallis, Pearson chi-square or for ICU/death 10df Wld test from multinomial logit. 
Table 2

Baseline and 2-day rate of change for biomarkers by COVID-19 subgroups identified using latent profile analysis.

\begin{tabular}{|c|c|c|c|c|c|c|c|c|c|c|}
\hline \multirow[t]{2}{*}{ Biomarker } & \multicolumn{2}{|c|}{ Class 1: Typical response } & \multicolumn{2}{|c|}{$\begin{array}{l}\text { Class } 2 \text { : Rapid } \\
\text { hyperinflammatory } \\
\text { response }\end{array}$} & \multicolumn{2}{|c|}{$\begin{array}{l}\text { Class 3: Progressive } \\
\text { inflammatory response }\end{array}$} & \multicolumn{2}{|c|}{$\begin{array}{l}\text { Class } 4 \text { : Inflammatory } \\
\text { response with renal injury }\end{array}$} & \multicolumn{2}{|c|}{$\begin{array}{l}\text { Class 5: Hyperinflammatory } \\
\text { response with renal injury }\end{array}$} \\
\hline & Baseline & 2-day $\Delta$ & Baseline & 2-day $\Delta$ & Baseline & 2-day $\Delta$ & Baseline & 2-day $\Delta$ & Baseline & 2-day $\Delta$ \\
\hline \multicolumn{11}{|l|}{ Physiological } \\
\hline $\begin{array}{l}\text { Respiratory rate, } \\
\text { /min }\end{array}$ & $\begin{array}{l}19.0[18.0 \\
20.0]\end{array}$ & $\begin{array}{l}-0.1[-1.3, \\
1.7]\end{array}$ & $\begin{array}{l}20.0[18.0, \\
22.0]\end{array}$ & $\begin{array}{l}0.5[-1.4 \\
2.3]\end{array}$ & $\begin{array}{l}20.0[18.0 \\
22.0]\end{array}$ & $\begin{array}{l}-0.0[-1.8, \\
1.9]\end{array}$ & $\begin{array}{l}19.0[18.0, \\
20.0]\end{array}$ & $0.1[-1.1,1.8]$ & $\begin{array}{l}20.0[18.0, \\
22.0]\end{array}$ & $\begin{array}{l}-0.1[-1.4, \\
1.9]\end{array}$ \\
\hline Oxygen saturation, \% & $\begin{array}{l}97.0[95.0, \\
98.0]\end{array}$ & $\begin{array}{l}-0.4[-1.1, \\
0.2]\end{array}$ & $\begin{array}{l}96.0[94.0, \\
97.0]\end{array}$ & $\begin{array}{l}-0.5[-1.2, \\
0.2]\end{array}$ & $\begin{array}{l}96.0[95.0, \\
97.0]\end{array}$ & $\begin{array}{l}-0.4[-1.1, \\
0.3]\end{array}$ & $\begin{array}{l}96.0[95.0, \\
98.0]\end{array}$ & $\begin{array}{l}-0.2[-0.7, \\
0.6]\end{array}$ & $\begin{array}{l}96.0[95.0, \\
98.0]\end{array}$ & $\begin{array}{l}-0.5[-1.4, \\
0.2]\end{array}$ \\
\hline Heart rate, /min & $\begin{array}{l}83.0[71.0, \\
90.0]\end{array}$ & $\begin{array}{l}-1.4[-7.0, \\
4.0]\end{array}$ & $\begin{array}{l}88.0[80.0 \\
100.0]\end{array}$ & $\begin{array}{l}-3.5[-11.4 \\
4.0]\end{array}$ & $\begin{array}{l}87.0[77.0 \\
97.0]\end{array}$ & $\begin{array}{l}-2.9[-8.8, \\
3.8]\end{array}$ & $\begin{array}{l}80.5[69.5, \\
90.0]\end{array}$ & $\begin{array}{l}-1.8[-9.1, \\
5.0]\end{array}$ & $\begin{array}{l}82.0[71.0 \\
92.0]\end{array}$ & $\begin{array}{l}-2.2[-10.5 \\
6.6]\end{array}$ \\
\hline Temperature, ${ }^{\circ} \mathrm{C}$ & $\begin{array}{l}36.9[36.6, \\
37.4]\end{array}$ & $\begin{array}{l}-0.0[-0.3, \\
0.3]\end{array}$ & $\begin{array}{l}36.8[36.6, \\
37.3]\end{array}$ & $\begin{array}{l}-0.1[-0.3 \\
0.3]\end{array}$ & $\begin{array}{l}37.1[36.7, \\
37.6]\end{array}$ & $\begin{array}{l}-0.1[-0.4, \\
0.2]\end{array}$ & $\begin{array}{l}37.0[36.6, \\
37.5]\end{array}$ & $\begin{array}{l}-0.1[-0.7 \\
0.3]\end{array}$ & $\begin{array}{l}36.7[36.5, \\
37.0]\end{array}$ & $\begin{array}{l}-0.0[-0.3 \\
0.3]\end{array}$ \\
\hline $\begin{array}{l}\text { Systolic blood } \\
\text { pressure, } \mathrm{mmHg}\end{array}$ & $\begin{array}{l}122.0[110.0, \\
135.0]\end{array}$ & $\begin{array}{l}-1.1[-6.7, \\
4.5]\end{array}$ & $\begin{array}{l}121.0 \text { [108.0, } \\
137.0]\end{array}$ & $\begin{array}{l}0.8[-5.2, \\
8.0]\end{array}$ & $\begin{array}{l}127.0 \text { [114.0, } \\
142.0]\end{array}$ & $\begin{array}{l}-1.1[-7.3, \\
4.9]\end{array}$ & $\begin{array}{l}133.5[118.5, \\
154.0]\end{array}$ & $\begin{array}{l}-3.4[-13.0, \\
3.5]\end{array}$ & $\begin{array}{l}121.0 \text { [107.5, } \\
133.0]\end{array}$ & $\begin{array}{l}4.6[-4.6 \\
12.1]\end{array}$ \\
\hline $\begin{array}{l}\text { Diastolic blood } \\
\text { pressure, } \mathrm{mmHg}\end{array}$ & $\begin{array}{l}70.0[62.0, \\
78.0]\end{array}$ & $\begin{array}{l}-0.3[-2.9, \\
2.4]\end{array}$ & $\begin{array}{l}70.0[61.0, \\
78.0]\end{array}$ & $\begin{array}{l}0.4[-4.0, \\
3.3]\end{array}$ & $\begin{array}{l}74.0[66.0, \\
84.0]\end{array}$ & $\begin{array}{l}-0.5[-4.0, \\
2.3]\end{array}$ & $\begin{array}{l}75.0[66.5, \\
89.5]\end{array}$ & $\begin{array}{l}-2.3[-6.5 \\
1.5]\end{array}$ & $\begin{array}{l}66.0[58.0, \\
77.5]\end{array}$ & $1.4[-3.3,5.4]$ \\
\hline \multicolumn{11}{|l|}{ Blood-based } \\
\hline WBC count, $\times 10^{9} / \mathrm{L}$ & $4.8[3.9,5.7]$ & $\begin{array}{l}-0.5[-1.0, \\
0.2]\end{array}$ & $\begin{array}{l}16.8 \text { [14.2, } \\
20.9]\end{array}$ & $\begin{array}{l}-2.9[-4.7, \\
-0.6]\end{array}$ & $8.3[7.2,9.7]$ & $\begin{array}{l}-0.9[-1.8, \\
-0.2]\end{array}$ & $5.0[3.7,6.1]$ & $\begin{array}{l}-0.5[-1.2, \\
-0.1]\end{array}$ & $9.4[7.9,11.1]$ & $\begin{array}{l}-1.2[-2.7 \\
-0.4]\end{array}$ \\
\hline $\begin{array}{l}\text { Lymphocyte count, } \\
\times 10^{9} / \mathrm{L}\end{array}$ & $0.9[0.6,1.2]$ & $\begin{array}{l}-0.1[-0.1, \\
0.0]\end{array}$ & $1.1[0.7,1.6]$ & $\begin{array}{l}-0.0[-0.2, \\
0.1]\end{array}$ & $1.1[0.8,1.5]$ & $\begin{array}{l}-0.0[-0.1, \\
0.1]\end{array}$ & $0.6[0.5,1.0]$ & $\begin{array}{l}-0.0[-0.1, \\
0.1]\end{array}$ & $0.9[0.6,1.3]$ & $\begin{array}{l}-0.0[-0.1, \\
0.0]\end{array}$ \\
\hline $\begin{array}{l}\text { Neutrophil count, } \\
\times 10^{9} / \mathrm{L}\end{array}$ & $3.4[2.5,4.1]$ & $\begin{array}{l}-0.4[-0.9, \\
0.3]\end{array}$ & $\begin{array}{l}13.5[12.1, \\
17.2]\end{array}$ & $\begin{array}{l}-2.1[-4.0, \\
-0.5]\end{array}$ & $6.5[5.5,7.8]$ & $\begin{array}{l}-0.8[-1.7, \\
0.1]\end{array}$ & $3.8[2.8,4.7]$ & $\begin{array}{l}-0.4[-0.9, \\
0.0]\end{array}$ & $7.9[6.4,9.4]$ & $\begin{array}{l}-1.0[-2.6, \\
0.2]\end{array}$ \\
\hline Urea, mmol/L & $5.6[4.0,8.5]$ & $\begin{array}{l}-0.7[-1.5 \\
-0.1]\end{array}$ & $9.0[6.0,12.6]$ & $\begin{array}{l}-0.6[-2.0 \\
0.2]\end{array}$ & $5.8[4.2,8.6]$ & $\begin{array}{l}-0.5[-1.3, \\
0.3]\end{array}$ & $\begin{array}{l}16.7[13.7, \\
23.9]\end{array}$ & $\begin{array}{l}-0.9[-3.7, \\
2.8]\end{array}$ & $\begin{array}{l}20.0[15.5, \\
28.6]\end{array}$ & $\begin{array}{l}-2.7[-7.3 \\
0.6]\end{array}$ \\
\hline Creatinine, umol/L & $\begin{array}{l}85.0[68.0, \\
107.0]\end{array}$ & $\begin{array}{l}-11.4 \\
{[-18.1,-6.1]}\end{array}$ & $\begin{array}{l}95.0[66.0, \\
152.0]\end{array}$ & $\begin{array}{l}-12.2 \\
{[-25.6,-4.4]}\end{array}$ & $\begin{array}{l}81.0[63.0, \\
101.0]\end{array}$ & $\begin{array}{l}-11.5 \\
{[-17.5,-5.1]}\end{array}$ & $\begin{array}{l}408.0[234.0, \\
692.0]\end{array}$ & $\begin{array}{l}-12.0 \\
{[-60.7,14.1]}\end{array}$ & $\begin{array}{l}210.0 \text { [164.0, } \\
298.0]\end{array}$ & $\begin{array}{l}-38.6[-76.2, \\
-14.6]\end{array}$ \\
\hline $\begin{array}{l}\text { C-reactive protein, } \\
\mathrm{mg} / \mathrm{L}\end{array}$ & $\begin{array}{l}45.6[20.2, \\
84.0]\end{array}$ & $\begin{array}{l}25.9[4.6, \\
45.6]\end{array}$ & $\begin{array}{l}133.3[79.4, \\
227.0]\end{array}$ & $\begin{array}{l}44.0[3.1, \\
82.4]\end{array}$ & $\begin{array}{l}94.0[48.5, \\
154.4]\end{array}$ & $\begin{array}{l}33.1[4.2, \\
79.1]\end{array}$ & $\begin{array}{l}62.6[32.1, \\
129.3]\end{array}$ & $\begin{array}{l}31.2[2.3 \\
83.4]\end{array}$ & $\begin{array}{l}\text { 133.1 [78.0, } \\
208.1]\end{array}$ & $\begin{array}{l}20.5[-19.0, \\
74.2]\end{array}$ \\
\hline Haemoglobin, g/L & $\begin{array}{l}128.0[112.0, \\
141.0]\end{array}$ & $\begin{array}{l}-9.6[-11.8, \\
-7.2]\end{array}$ & $\begin{array}{l}126.0 \text { [106.0, } \\
140.0]\end{array}$ & $\begin{array}{l}-11.2[-14.7, \\
-8.7]\end{array}$ & $\begin{array}{l}131.0 \text { [118.0, } \\
142.0]\end{array}$ & $\begin{array}{l}-10.1 \\
{[-12.2,-8.1]}\end{array}$ & $\begin{array}{l}104.0[90.0, \\
116.0]\end{array}$ & $\begin{array}{l}-7.0[-9.7 \\
-5.3]\end{array}$ & $\begin{array}{l}121.0[105.0, \\
136.0]\end{array}$ & $\begin{array}{l}-9.9[-13.9, \\
-6.7]\end{array}$ \\
\hline Platelet count, $\times 10^{9} / \mathrm{L}$ & $\begin{array}{l}186.0[145.0, \\
234.0]\end{array}$ & $\begin{array}{l}-13.0 \\
{[-31.2,3.4]}\end{array}$ & $\begin{array}{l}279.0 \text { [201.0, } \\
393.0]\end{array}$ & $\begin{array}{l}-18.6 \\
{[-46.7,15.1]}\end{array}$ & $\begin{array}{l}239.0 \text { [184.0, } \\
303.0]\end{array}$ & $\begin{array}{l}-1.7[-22.4, \\
22.4]\end{array}$ & $\begin{array}{l}159.0 \text { [118.0, } \\
223.0]\end{array}$ & $\begin{array}{l}-18.6 \\
{[-35.5,-5.0]}\end{array}$ & $\begin{array}{l}219.0 \text { [154.0, } \\
271.0]\end{array}$ & $\begin{array}{l}-14.2[-35.9, \\
-1.0]\end{array}$ \\
\hline Albumin, g/L & $\begin{array}{l}37.0[34.0, \\
40.0]\end{array}$ & $\begin{array}{l}-4.8[-5.5, \\
-3.8]\end{array}$ & $\begin{array}{l}34.0[30.0, \\
37.5]\end{array}$ & $\begin{array}{l}-5.3[-6.8, \\
-4.0]\end{array}$ & $\begin{array}{l}37.0[34.0, \\
40.0]\end{array}$ & $\begin{array}{l}-4.9[-5.6 \\
-4.0]\end{array}$ & $\begin{array}{l}34.0[30.0, \\
37.0]\end{array}$ & $\begin{array}{l}-4.8[-5.5 \\
-3.9]\end{array}$ & $\begin{array}{l}33.0[30.0, \\
37.0]\end{array}$ & $\begin{array}{l}-5.4[-7.0, \\
-4.1]\end{array}$ \\
\hline
\end{tabular}

Data are presented as median [IQR].

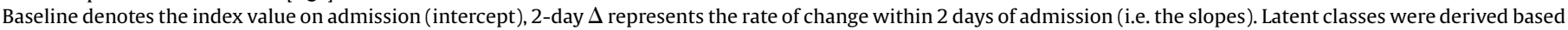
from both the intercept and slopes (in a single model).

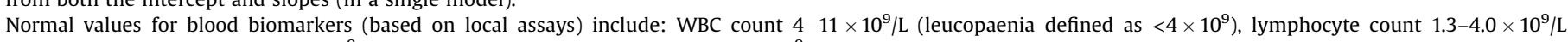

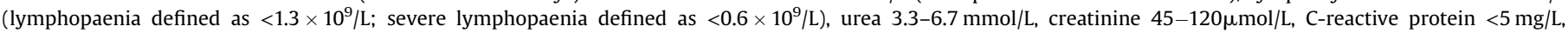

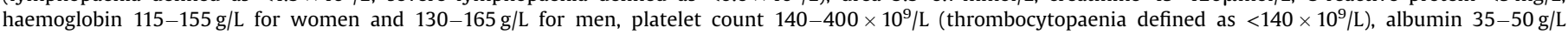
(hypoalbuminaemia defined as $<35 \mathrm{~g} / \mathrm{L}$ ).

(median $1.1 \times 10^{9} / \mathrm{L}$ [IQR $\left.0.7,1.6\right]$ for Class 2 versus $0.9 \times 10^{9} / \mathrm{L}$ [IQR $0.6,1.2$ ] for Class $1, \mathrm{p}=\mathrm{NS}$ ). Within the first two days, these patients showed the fastest rising CRP and fastest declining white cell and neutrophil counts, though all remained above clinically normal values. Haemoglobin decreased. The 2-day trajectory of lymphocyte count was similar to the typical response (median $\Delta$ $-0.0 \times 10^{9} / \mathrm{L}[\mathrm{IQR}-0.2,0.1]$ for Class 2 versus $-0.1 \times 10^{9} / \mathrm{L}[\mathrm{IQR}$ $-0.1,0.0$ ] for Class $1, \mathrm{p}=\mathrm{NS}$ ), as was the evolution of platelet count (median $\Delta-18.6 \times 10^{9} / \mathrm{L}[-46.7,15.1]$ for Class 2 versus $-13.0 \times 10^{9} / \mathrm{L}$ $[-31.2,3.4]$ for Class $1, \mathrm{p}=.011$. Compared with the typical response, Class 2 patients were older (median age 73 years), predominantly White (76\%), and with moderate prevalence of comorbidities (hypertension $52 \%$, diabetes $30 \%$ ). Class 2 had the highest likelihood of death within the first two days of admission and days 3-14 (38\% and 8\% respectively).

Class 3 (18\%, progressive inflammatory response) displayed neutrophilia (with normal-range white cell and lymphocyte counts) and raised CRP. Compared to the typical response, patients in Class 3 had higher CRP $(\mathrm{p}<0.001)$ and infection response markers (white cell count and neutrophils, lymphocytes; all $\mathrm{p}<0.001$ ). Within the first two days, trajectories of all biomarkers were similar to the typical response, with the exception of a lesser drop in platelet count (median $\Delta-1.7 \times 10^{9} / \mathrm{L}$ [IQR $\left.-22.4,22.4\right]$ for Class 3 versus $-13.0 \times 10^{9} / \mathrm{L}$ [IQR $\left.-31.2,3.4\right]$ for Class $\left.1, \mathrm{p}<0.001\right)$. This group had a similar distribution of comorbidities as Class 1 , but a higher likelihood of being transferred to ICU within the first two days of admission (4\%) and in days 3-14 (8\%). Death rates for this group were not significantly higher than for Class 1 within the first two days of admission (3\%) and days 3-14 (15\%).

Class 4 (6\%, inflammatory response with renal injury) was characterised by severe renal failure (median urea $16.7 \mathrm{mmol} / \mathrm{L}$ and creatinine $408 \mathrm{umol} / \mathrm{L}$ ), anaemia, prominent lymphopaenia (the lowest among all classes) and hypoalbiminaemia. Compared with the typical COVID19 response, this group had sligthly higher baseline CRP but significantly lower lymphocyte count $(\mathrm{p}<0.001$ for both). Neutrophil counts and haemoglobin were also significantly lower $(\mathrm{p}<0.001)$; with haemoglobin below clinically normal levels for men and women. Within the first two days, Class 4 patients showed slowly improving creatinine (though still clinically abnormal) and worsening anaemia. Compared to Class 1, these patients were more likely to be male (68\%), BAME descent (66\%), and had the highest comorbidity burden among classes (hypertension $81 \%$, IHD $21 \%$, heart failure $19 \%$, diabetes $64 \%$ and CKD 69 \%).

Finally, Class 5 (9\%, hyperinflammatory response with renal injury) was also characterised by renal failure (median urea $20.0 \mathrm{mmol} / \mathrm{L}$ and creatinine $210 \mathrm{umol} / \mathrm{L}$ ), anaemia, hypoalbuminaemia, lymphopaenia and elevated neutrophil count. Median creatinine levels were lower than for Class 4 and haemoglobin levels were higher, suggesting less chronic and severe of renal 


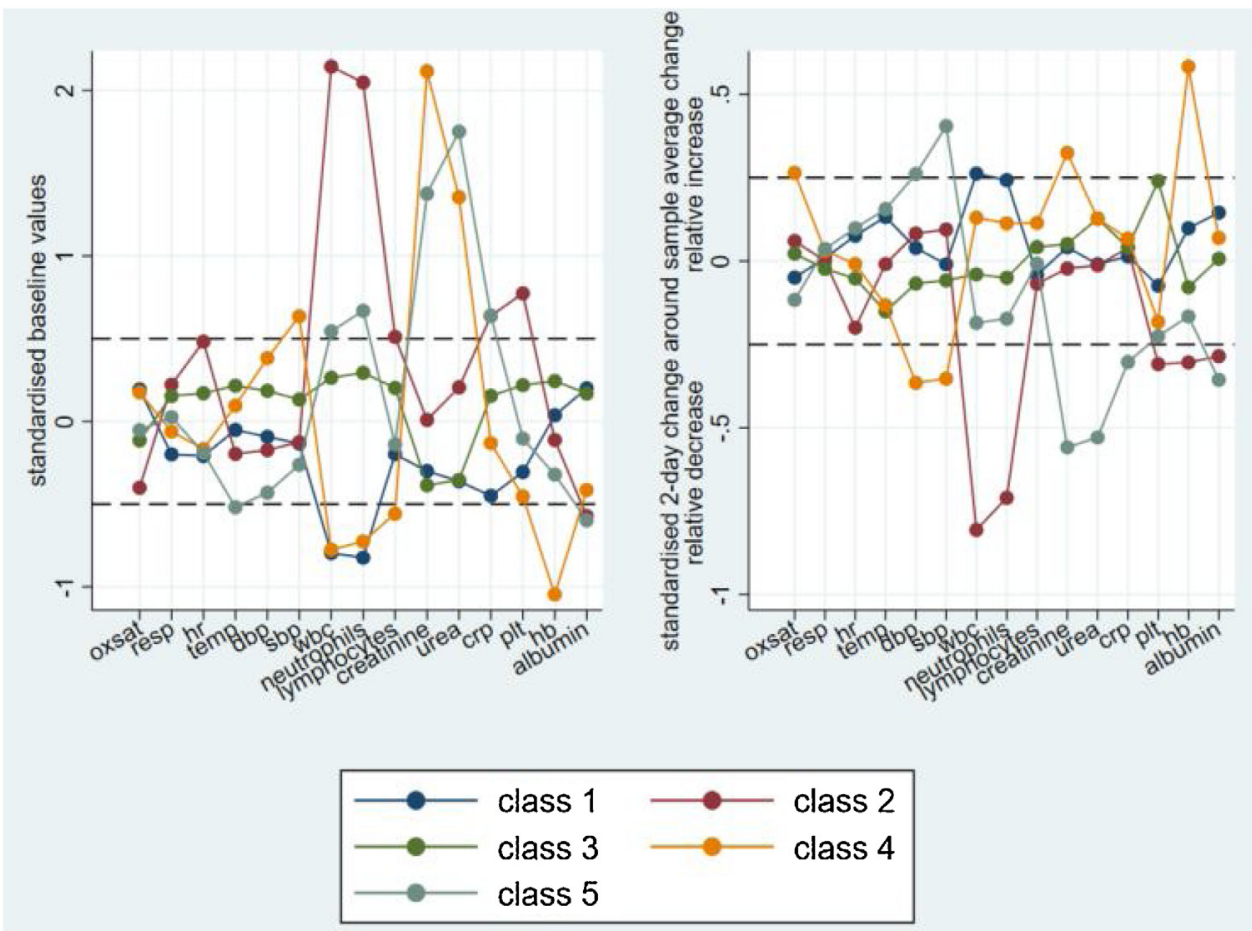

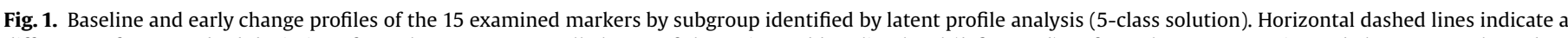

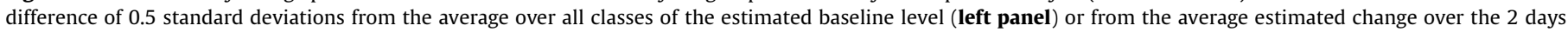
following admission (right panel).

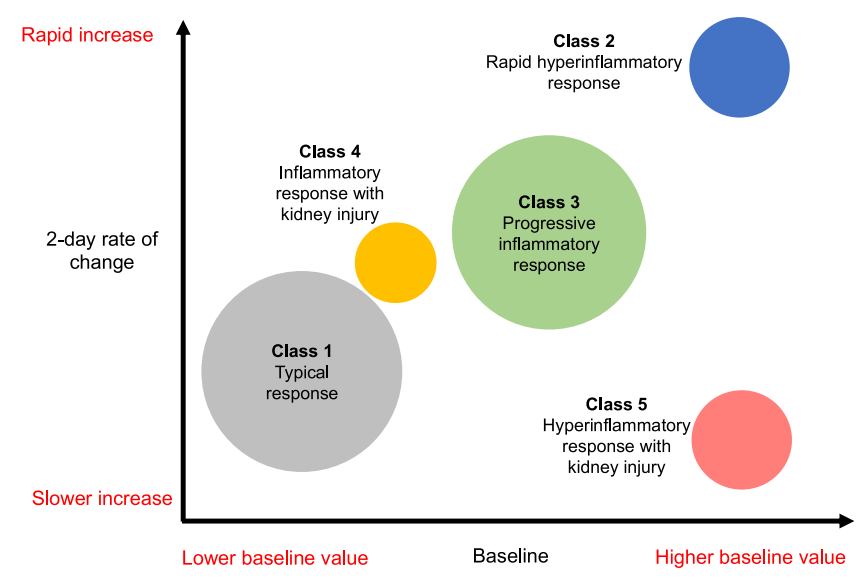

Fig. 2. Schematic diagram illustrating baseline (admission) values and trajectories of C-reactive protein by class. Circles represent classes identified by latent profile analysis, with a cross-sectional area proportional to the number of patients in each group and position on the diagram determined by the relative values of baseline $C$ reactive protein (CRP) on the $x$-axis and 2-day rate of change on the $y$-axis (the scale representing the minimum to maximum observed among all groups). *Acute kidney injury was defined according to the Kidney Disease: Improving Global Outcomes definition [11].

failure. The rise in CRP was much more attenuated in this group compared with the other 'hyperinflammatory' Class 2. Compared with Class 1 (typical response), they exhibited more severe infection response (higher white cell, neutrophil and platelet counts; all $p<0.01$ ) and lower haemoglobin ( $p=0.042)$. Within the first two days of admission, this group showed the fastest recovery rate of serum creatinine, although levels remained clinically abnormally. Patients in Class 5 were older than other classes (median age 78 years), predominantly male (70\%) and White ethnicity (61\%). Comorbidity burden was high and varied in this group (COPD $19 \%$, hypertension $73 \%$, CKD $49 \%$, diabetes $44 \%$, IHD $23 \%$ ). Compared with Class 1 these patients had higher death rates within the first two days of admission (8\%) and in days 3-14 (37\%).

\section{Association between classes and adverse in-hospital outcomes}

Within the first two days, 39 of 1335 (3\%) patients were admitted to ICU and 45 (3\%) died without ICU admission. Beyond 2 days, among 1251 patients who were alive and not admitted to ICU, 66 patients (5.2\%) were subsequently admitted to ICU and 228 patients $(18.2 \%)$ died in hospital within 14 days. Outcomes are summarised in Table 1. Kaplan Meier survival curves are shown in Supplemental Figure 8.

Latent profile membership was associated with outcomes in days $0-2$ and between 3-14 days (Table 3 ). These associations were attenuated when adjusted for demographics (Model 3) and comorbidities (Model 4). Nonetheless, differences by latent profile remained $(\mathrm{p}<.001)$ with both Classes 2 and 5 showing elevated risk of death or a combined endpoint of death or ICU admission.

\section{Discussion}

We have identified five distinct classes of biological response to COVID-19, based on early physiological and blood biomarker profiles during hospitalisation. All classes displayed evidence of inflammation, to varying degrees, and included patients across the spectrum of demographic and comorbidity characteristics, with some preferential distribution of older age (Classes 2 and 5) and comorbidity burden (Classes 4 and 5). Notably, early physiological (clinical) markers, including respiratory rate and oxygen saturation, although used in the classification technique, were not ultimately different between classes. However, differences in mortality rates were observed between classes. Therefore, these data demonstrate heterogeneous patterns of biological response to 
Table 3

Multinomial logit regression analysis of mortality risk across COVID-19 subgroups.

\begin{tabular}{|c|c|c|c|c|c|}
\hline Outcome & $\begin{array}{l}\text { Model } 1 \\
\text { RRR (95 \% CI) }\end{array}$ & $\begin{array}{l}\text { Model } 2 \\
\text { RRR (95 \% CI) }\end{array}$ & $\begin{array}{l}\text { Model } 3 \\
\text { RRR (95 \% CI) }\end{array}$ & $\begin{array}{l}\text { Model } 4 \\
\text { RRR (95 \% CI) }\end{array}$ & $\begin{array}{l}\text { Model } 5 \\
\text { RRR (95 \% CI) }\end{array}$ \\
\hline $\begin{array}{l}\text { Death* } \\
\text { Class1 }\end{array}$ & $\begin{array}{l}\mathrm{p}=.004 \\
\text { reference }\end{array}$ & $\mathrm{p}<.001$ & $\mathrm{p}<.001$ & $\mathrm{p}<.001$ & $\mathrm{p}=.017$ \\
\hline Class2 & $7.67(2.73,21.55)$ & $3.63(2.24,5.86)$ & $3.21(1.93,5.33)$ & $3.20(1.91,5.36)$ & $2.28(1.33,3.93)$ \\
\hline Class3 & $3.06(1.20,7.83)$ & $1.26(0.87,1.81)$ & $1.29(0.88,1.89)$ & $1.30(0.89,1.92)$ & $1.11(0.74,1.65)$ \\
\hline Class 4 & $2.23(0.44,11.30)$ & $1.77(0.94,3.32)$ & $1.82(0.95,3.51)$ & $1.58(0.78,3.21)$ & $1.33(0.64,2.75)$ \\
\hline Class5 & $7.39(2.63,20.77)$ & $3.78(2.37,6.04)$ & $2.70(1.65,4.40)$ & $2.40(1.43,4,01)$ & $1.79(1.05,3.05)$ \\
\hline $\begin{array}{l}\text { Death or ICU* } \\
\text { Class1 }\end{array}$ & $\begin{array}{l}\mathrm{p}<.001 \\
\text { reference }\end{array}$ & $\mathrm{p}<.001$ & $\mathrm{p}<.001$ & $\mathrm{p}<.001$ & $\mathrm{p}<.027$ \\
\hline Class2 & $4.93(2.31,10.52)$ & $3.30(2.10,5.18)$ & $3.07(1.93,4.87)$ & $3.06(1.92,4.87)$ & $2.18(1.33,3.55)$ \\
\hline Class3 & $2.86(1.52,5.35)$ & $1.48(1.07,2.03)$ & $1.52(1.09,2.10)$ & $1.52(1.10,2.12)$ & $1.26(0.90,1.78)$ \\
\hline Class 4 & $2.88(1.07,7.72)$ & $1.85(1.05,3.25)$ & $1.77(1.00,3.14)$ & $1.56(0.84,2.90)$ & $1.31(0.69,2.47)$ \\
\hline Class5 & $3.80(1.71,8.45)$ & $3.11(1.99,4.86)$ & $2.40(1.52,3.80)$ & $2.18(1.35,3.52)$ & $1.63(0.99,2.69)$ \\
\hline
\end{tabular}

RRR, relative risk ratio.

Model 1 - Outcomes within 0-2 days, unadjusted.

Model 2 - Outcomes between 3-14 days, unadjusted.

Model 3 - Outcomes between 3-14 days, adjusted for age and sex.

Model 4 - Outcomes between 3-14 days, adjusted for age, sex and pre-existing comorbidities.

Model 5 - Outcomes between 3-14 days, adjusted for age, sex, pre-existing comorbidities, and baseline NEWS2 score.

p-values derived using Wald test with 5 degrees of freedom, compared with Class 1 (reference class).

COVID-19 with different disease trajectories, which may have prognostic and therapeutic relevance.

The typical COVID-19 biological response in our cohort (38\% of patients), displayed a moderate and rising CRP, and mild and stable lymphopaenia in the early hospitalisation period. These features have been noted in previous COVID-19 studies [2,4,5,12]. Several studies have also reported thrombocytopaenia on presentation, though it's ability to discriminate between mild and moderate versus severe or critical disease, may not be as robust or consistent as lymphopaenia [12-15]. In our cohort, there was some variation in absolute platelet counts between classes, however admission values were within a normal physiological range for all classes, and only fell below normal at 2 days in Class 4 . Inflammation has been strongly implicated in the pathogenesis of COVID-19, and all classes in our study displayed evidence of an inflammatory response (i.e. elevated CRP), which increased further in the early admission period. Two groups, however, had greater than typical levels of inflammation (Classes 2 and 5) corresponding with a greater risk of in-hospital mortality. Both of these hyperinflammatory groups included patients who were significantly older than average and Class 2 exhibited greater neutrophilia than the typical response. A strong association with older age has been noted in nearly all studies of severe COVID-19 [2-5]. Ageing is also reportedly associated with a chronic low grade inflammatory state [16] and impaired innate and adaptive immunity ('immunosenescence') [17]. A severe uncontrolled inflammatory response, possibly heralded by early neutrophil engagement, may be one mechanism of adverse outcomes among older patients with COVID-19.

We also identified two groups characterised by renal failure (Classes 4 and 5) who had discordant white cell and inflammatory responses. Class 4 had lower white cell counts (lymphocytes and neutrophils) and an attenuated rise in CRP compared with Class 5. Several factors may contribute to these group differences. Class 4 contained more individuals from BAME groups than Class 5 (66\% versus $39 \%$ ) and familial reductions in white cell and neutrophil counts are widely recognised in people of African ancestry [18,19]. Conversely, patients in class 5 were, on average, older, and agerelated immunosenscence may have predisposed class 5 individuals to a heightened inflammatory response. Both classes 4 and 5 had higher levels of inflammation than the typical response (Class 1 ), and a greater prevalence of comorbidity. Since Class 4 and Class 1 (the typical response) had a similar age distribution and symptom duration, we may infer that the excess comorbidity burden in Class 4 explained the increased inflammatory response to COVID-19. Systemic persistent inflammation is commonly seen in patients with end-stage renal disease who are uraemic, and such mechanisms may be further activated by superimposed infection such as COVID-19 [20] This may be a direct association (e.g. worse endothelial dysfunction), indirect via polypharmacy, or comorbidity burden may be a surrogate for fraily-related immune dysfunction. However, when comparing Class 2 and Class 5 (two groups of similar age and ethnicity but unequal prevalence of comorbidities) we do not observe a significant difference in inflammatory response. Thus, additional unrecognised factors likely influence the tendency towards hyperinflammation.

Class 3 patients had broadly similar biomarker profiles, clinical characteristics, and mortality risk as the typical response, with the exception of a greater and steeper trajectory of inflammatory response. Although we have labelled these patients as having more progressive COVID-19 disease, they also presented slightly later to hospital than Class 1, which may be a relevant confounder. The lower 2-day decline in platelet count as compared with Class 1 , may refelect the initial stages of longitudinal recovery in this biomarker, as has been noted in other studies [12]. The observation that neutrophil and CRP counts remain high or increase further in this group, as compared with the typical response, may reflect discordant platelet and inflammatory responses or possible secondary (bacterial) infection contributing to infection severity.

It is striking that clinical (physiological) markers, which are often relied on for clinical risk stratification in the acute setting, did not vary significantly between classes of COVID-19 response, nor were they clinically abnormal. This mirrors anecdotal observations that systemic haemodynamic changes are infrequent in the early stages of COVID-19, and suggests that in the early hospitalisation period, additional relevant information can be gained from serial measurement of selected blood biomarkers. The absolute changes in biomarker values were relatively small, compared with those seen in non-COVID pneumonia or sepsis [21], nevertheless there were clinically meaningful differences between classes.

Although the aim of our study was not risk stratification, we observed different mortality rates between the classes. This supports the notion that biomarker-defined classes could reflect prognostically-relevant mechanisms of COVID-19 pathophysiology, that would benefit from stratified approaches to therapy. Many existing COVID-19 clinical trials have intentionally broad enrollment criteria, designed to capture the full public health impact of the disease [22]. However, the use of targeted therapies may be more effective in patient subgroups with biologically similar responses and 
information from biomarker trajectories may inform the design and timing of application of new therapies for COVID-19.

Strengths of our study include the large cohort and assessment of serial measures beyond baseline, which enable more comprehensive characterisation of hospitalised patients with COVID-19 and their disease course. The analytical approach used allows us to capture the complexity and heterogeneity of biological responses as it accounts for between and within-person variability (that is, it accounts for the fact that each patient is different at baseline and has unique rates of change over time). Although our findings can be drivers for hypothesis-generation, causality cannot be ascertained and external validation is required. Some other limitations should be acknowledged. Data regarding ICU admission must be considered within the context of clinical decision making regarding eligibility versus ceilings of care for frail individuals. Although we selected a parsimonious model of routinely collected biomarkers that cover a wide range of potential biological processes, other blood parameters (e.g., such as troponin, Ddimers and ferritin) were not included due to missingness or lack of repeated testing. Future studies should aim to explore these additional biomarkers to further our understanding of COVID-19 pathophysiology. Our data were derived from patients admitted to hospital with COVID19 and who had biomarker data available up to and including 14 days. This study did not examine the clustering of complications or organ-specific dysfunction, such as thromboembolism, cardiovascular or cerebrovascular ischaemia. These outcomes typically occur later in the course of severe disease, outside of the window in which our models were based. Additional research is needed to establish whether these complications may influence clustering or be differentially distributed across classes. Further research is also needed to explore ethnic variation in biomarker data, which may have influenced the clinical phenotypes in this study.

\section{Conclusions}

We have provided empirical evidence of potentially distinct biological responses to COVID-19 in hospitalised patients, based on the trajectories of physiological and blood biomarkers. These classes may indicate different (dominant) mechanisms of disease that warrant stratified approaches to COVID-19 therapy. In particular, further research is needed to determine whether patients with early hyperinflammatory responses and kidney injury warrant earlier or specific inflammation-targeted intervention(s) in order to improve their in-hospital outcomes.

\section{Ethics approval and consent to participate}

This project operated under London South East Research Ethics Committee approval (reference 18/LO/2048) granted to the King's Electronic Records Research Interface (KERRI). Given the current context, specific work on COVID19 research was reviewed with a virtually convened 4-expert patient panel input on a virtual committee with Caldicott Guardian oversight. In addition, the KERRI database used in this study was developed according to $\mathrm{KCH}$ use of data for research guidelines (https://www.kch.nhs.uk/ research/use-of-data-for-research) and patient engagement groups for this dataset have been conducted in 2018-2019. The KERRI approvals allow the processing of data on the basis of optout not written informed consent as per the Research Ethics approvals granted by the Health Research Authority which is the UK national regulator of health research.

\section{Consent for publication}

Not applicable

\section{Availability of data and materials}

Source text from patient records used in the study will not be available due to inability to fully anonymise up to the Information Commissioner Office (ICO) standards and would be likely to contain strong identifiers (e.g. names, postcodes) and highly sensitive data (e.g. diagnoses). A subset of the dataset limited to anonymisable information (e.g. only SNOMED codes and demographics) is available on request to researchers with suitable training in information governance and human confidentiality protocols subject to approval by the King's College Hospital Information Governance committee; applications for research access should be sent to kch-tr.cogstackrequests@nhs.net. This dataset cannot be released publicly due to the risk of reidentification of such granular individual level data, as determined by the King's College Hospital Caldicott Guardian.

\section{Declaration of Competing Interest}

JTHT received research support and funding from InnovateUK, Bristol-Myers-Squibb, iRhythm Technologies, and holds shares $<£ 5000$ in Glaxo Smithkline and Biogen. All other authors declare that they have no competing interests.

\section{Funding}

RZ is supported by a King's Prize Fellowship and the British Heart Foundation Centre for Cardiovascular Research Excellence at King's College London.

DMB is funded by a UKRI Innovation Fellowship as part of Health Data Research UK MR/S00310X/1 (https://www.hdruk.ac. $\mathrm{uk})$.

KO'G is supported by an MRC Clinical Training Fellowship (MR/ R017751/1).

AS is supported by a King's Medical Research Trust studentship. AMS is supported by the British Heart Foundation $(\mathrm{CH} /$ 1999001/11735), the National Institute for Health Research (NIHR) Biomedical Research Centre at Guy's \& St Thomas' NHS Foundation Trust and King's College London (IS-BRC-1215-20006), and the Fondation Leducq.

AP is partially supported by NIHRNF-SI-0617-10120 and NIHR Biomedical Research Centre at South London and Maudsley NHS Foundation Trust and King's College London.

RJBD is supported by: (1) NIHR Biomedical Research Centre at South London and Maudsley NHS Foundation Trust and King's College London, London, U.K. (2) Health Data Research UK, which is funded by the UK Medical Research Council, Engineering and Physical Sciences Research Council, Economic and Social Research Council, Department of Health and Social Care (England), Chief Scientist Office of the Scottish Government Health and Social Care Directorates, Health and Social Care Research and Development Division (Welsh Government), Public Health Agency (Northern Ireland), British Heart Foundation and Wellcome Trust. (3) The BigData@Heart Consortium, funded by the Innovative Medicines Initiative-2 Joint Undertaking under grant agreement No. 116074. This Joint Undertaking receives support from the European Union's Horizon 2020 research and innovation programme and EFPIA; it is chaired by DE Grobbee and SD Anker, partnering with 20 academic and industry partners and ESC. (4) The National Institute for Health Research University College London Hospitals Biomedical Research Centre. (5) National Institute for Health Research (NIHR) Biomedical Research Centre at South London and Maudsley NHS Foundation Trust and King's College London. (5) The UK Research and Innovation London Medical Imaging \& Artificial Intelligence Centre for Value Based Healthcare 
$\mathrm{RB}$ is funded in part by grant MR/R016372/1 for the King's College London MRC Skills Development Fellowship programme funded by the UK Medical Research Council (MRC, https://mrc.ukri. org) and by grant IS-BRC-1215-20018 for the National Institute for Health Research (NIHR, https://www.nihr.ac.uk) Biomedical Research Centre at South London and Maudsley NHS Foundation Trust and King's College London.

This paper represents independent research part-funded by the National Institute for Health Research (NIHR) Biomedical Research Centres at South London and Maudsley NHS Foundation Trust, London AI Medical Imaging Centre for Value-Based Healthcare, and Guy's \& St Thomas' NHS Foundation Trust, both with King's College London. The views expressed are those of the author(s) and not necessarily those of the NHS, the NIHR or the Department of Health and Social Care. The funders had no role in study design, data collection and analysis, decision to publish, or preparation of the manuscript.

\section{Authors' contributions}

RZ, AP, EC and RB conceived the study. RZ, AP, EC, DMB and RB participated in the study design. RZ, AP, EC, DMB, KOG, ZK, TS, AS, JG, JTHT, AS, RJBD, and RB participated in the data collection. AP and EC performed data analyses. RZ, AP, EC, DMB, KOG, ZK, TS, AS, JG, JTHT, AS, RJBD, and RB, contributed to data interpretation. RZ, $A P, E C$ and RB drafted the first version of the manuscript. All authors contributed to and approved the final manuscript and the decision to submit. The corresponding author attests that all listed authors meet authorship criteria and that no others meeting the criteria have been omitted.

\section{Disclaimer}

The views expressed are those of the authors and not necessarily those of the MRC, NHS, the NIHR or the Department of Health and Social Care. The funders of the study had no role in the study design, data collection, data analysis, data interpretation, writing of the report or the decision to submit the article for publication.

\section{Acknowledgements}

We would like to thank all the clinicians managing the patients, the patient experts of the KERRI committee, Professor Irene Higginson, Professor Alastair Baker, Professor Jules Wendon, Dan Persson and Damian Lewsley for their support.

The authors acknowledge use of the research computing facility at King's College London, Rosalind (https://rosalind.kcl.ac.uk), which is delivered in partnership with the National Institute for Health Research (NIHR) Biomedical Research Centres at South London \& Maudsley and Guy's \& St. Thomas' NHS Foundation Trusts, and part-funded by capital equipment grants from the Maudsley Charity (award 980) and Guy's \& St. Thomas' Charity (TR130505). The views expressed are those of the author(s) and not necessarily those of the NHS, the NIHR, King's College London, or the Department of Health and Social Care.

\section{Appendix A. Supplementary data}

Supplementary material related to this article can be found, in the online version, at doi:https://doi.org/10.1016/j. retram.2021.103276.

\section{References}

[1] Richardson S, Hirsch JS, Narasimhan M, et al. Presenting characteristics, comorbidities, and outcomes among 5700 patients hospitalized with COVID19 in the New York City area. JAMA 2020.

[2] Huang C, Wang Y, Li X, et al. Clinical features of patients infected with 2019 novel coronavirus in Wuhan, China. Lancet 2020;395(10223):497-506.

[3] Onder G, Rezza G, Brusaferro S. Case-fatality rate and characteristics of patients dying in relation to COVID-19 in Italy. JAMA 2020.

[4] Zhou F, Yu T, Du R, et al. Clinical course and risk factors for mortality of adult inpatients with COVID-19 in Wuhan, China: a retrospective cohort study. Lancet 2020;395(10229):1054-62.

[5] Wang D, Hu B, Hu C, et al. Clinical characteristics of 138 hospitalized patients with 2019 novel coronavirus-infected pneumonia in Wuhan, China. JAMA 2020.

[6] Tjendra Y, Al Mana AF, Espejo AP, et al. Predicting disease severity and outcome in COVID-19 patients: a review of multiple biomarkers. Arch Pathol Lab Med 2020.

[7] Calfee CS, Delucchi K, Parsons PE, et al. Subphenotypes in acute respiratory distress syndrome: latent class analysis of data from two randomised controlled trials. Lancet Respir Med 2014;2(8):611-20.

[8] Jackson R, Kartoglu I, Stringer C, et al. CogStack - experiences of deploying integrated information retrieval and extraction services in a large National Health Service Foundation Trust hospital. BMC Med Inform Decis Mak 2018;18 (1):47.

[9] Kraljevic Z, Bean D, Mascio A, et al. MedCAT - medical concept annotation tool. Preprint: arXiv:191210166. 2019.

[10] Bean DM, Kraljevic Z, Searle T, et al. ACE-inhibitors and Angiotensin-2 Receptor Blockers are not associated with severe SARS-COVID19 infection in a multi-site UK acute Hospital Trust. Eur J Heart Fail 2020

[11] Kellum J, Lameire N, Aspelin P, Barsoum RS, Burdmann EA, Goldstein SL, et al. Kidney disease: improving global outcomes (KDIGO) acute kidney injury work group. KDIGO clinical practice guideline for acute kidney injury. Kidney Int Suppl (2011) 2012;2(1):1-138.

[12] Chen R, Sang L, Jiang M, et al. Longitudinal hematologic and immunologic variations associated with the progression of COVID-19 patients in China. J Allergy Clin Immunol 2020;146(1):89-100.

[13] Lippi G, Plebani M, Henry BM. Thrombocytopenia is associated with severe coronavirus disease 2019 (COVID-19) infections: a meta-analysis. Clin Chim Acta 2020;506:145-8.

[14] Henry BM, de Oliveira MHS, Benoit S, Plebani M, Lippi G. Hematologic, biochemical and immune biomarker abnormalities associated with severe illness and mortality in coronavirus disease 2019 (COVID-19): a meta-analysis. Clin Chem Lab Med 2020;58(7):1021-8.

[15] Amgalan A, Othman M. Hemostatic laboratory derangements in COVID-19 with a focus on platelet count. Platelets 2020;31(6):740-5.

[16] Franceschi C, Bonafe M, Valensin S, et al. Inflamm-aging. An evolutionary perspective on immunosenescence. Ann N Y Acad Sci 2000;908:244-54.

[17] Aw D, Silva AB, Palmer DB. Immunosenescence: emerging challenges for an ageing population. Immunology 2007;120(4):435-46.

[18] Shaper AG, Lewis P. Genetic neutropenia in people of African origin. Lancet 1971;2(7732):1021-3.

[19] Grann VR, Bowman N, Joseph C, et al. Neutropenia in 6 ethnic groups from the Caribbean and the U.S. Cancer 2008;113(4):854-60.

[20] Cobo G, Lindholm B, Stenvinkel P. Chronic inflammation in end-stage renal disease and dialysis. Nephrol Dial Transplant 2018;33(suppl_3):iii35-40.

[21] Kellum JA, Kong L, Fink MP, et al. Understanding the inflammatory cytokine response in pneumonia and sepsis: results of the Genetic and Inflammatory Markers of Sepsis (GenIMS) Study. Arch Intern Med 2007;167(15):1655-63.

[22] Oxford. Uo. Randomised evaluation of COVID-19 therapy (recovery) trial. Published; 2020. . Accessed 28/06/2020 https://clinicaltrials.gov/ct2/show/ NCT04381936. 